\title{
An extreme ultraviolet Michelson interferometer for experiments at free-electron lasers
}

Vinzenz Hilbert, Alexander Blinne, Silvio Fuchs, Torsten Feigl, Tino Kämpfer, Christian Rödel, Ingo Uschmann, Martin Wünsche, Gerhard G. Paulus, Eckhart Förster, and Ulf Zastrau

Citation: Review of Scientific Instruments 84, 095111 (2013); doi: 10.1063/1.4821146

View online: https://doi.org/10.1063/1.4821146

View Table of Contents: http://aip.scitation.org/toc/rsi/84/9

Published by the American Institute of Physics

\section{Articles you may be interested in}

Spatio-temporal coherence of free-electron laser radiation in the extreme ultraviolet determined by a Michelson interferometer

Applied Physics Letters 105, 101102 (2014); 10.1063/1.4895455

A Fourier transform spectrometer without a beam splitter for the vacuum ultraviolet range: From the optical design to the first UV spectrum

Review of Scientific Instruments 80, 043101 (2009); 10.1063/1.3111452

Sensitivity calibration of an imaging extreme ultraviolet spectrometer-detector system for determining the efficiency of broadband extreme ultraviolet sources

Review of Scientific Instruments 84, 023101 (2013); 10.1063/1.4788732

A Michelson-type radio interferometer for university education

American Journal of Physics 84, 249 (2016); 10.1119/1.4940212

Design and test of a broadband split-and-delay unit for attosecond XUV-XUV pump-probe experiments

Review of Scientific Instruments 87, 023106 (2016); 10.1063/1.4941722

Circular grating interferometer for mapping transverse coherence area of X-ray beams

Applied Physics Letters 105, 041116 (2014); 10.1063/1.4892002

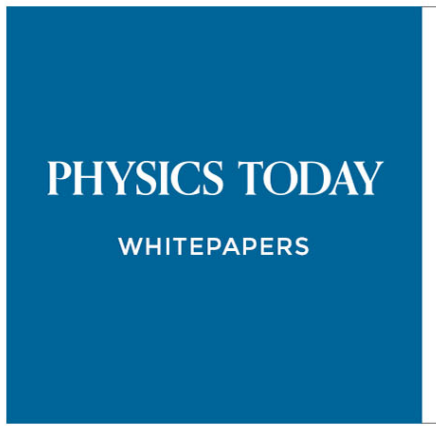

MANAGER'S GUIDE

Accelerate R\&D with

Multiphysics Simulation
READ NOW

PRESENTED BY

○OMSOL 


\title{
An extreme ultraviolet Michelson interferometer for experiments at free-electron lasers
}

\author{
Vinzenz Hilbert, ${ }^{1, a)}$ Alexander Blinne, ${ }^{2}$ Silvio Fuchs, ${ }^{1}$ Torsten Feigl, ${ }^{3}$ Tino Kämpfer, ${ }^{1,4}$ \\ Christian Rödel, ${ }^{1,4}$ Ingo Uschmann, ${ }^{1,4}$ Martin Wünsche, ${ }^{1,4}$ Gerhard G. Paulus, ${ }^{1}$ \\ Eckhart Förster, ${ }^{1,4}$ and Ulf Zastrau ${ }^{1}$ \\ ${ }^{1}$ Institute of Optics and Quantum Electronics, Friedrich-Schiller University Jena, Max-Wien-Platz 1, \\ 07743 Jena, Germany \\ ${ }^{2}$ Institute for Theoretical Physics, Friedrich-Schiller University Jena, Max-Wien-Platz 1, 07743 Jena, Germany \\ ${ }^{3}$ Fraunhofer Institute for Applied Optics and Precision Engineering, Albert-Einstein-Straße 7 , \\ 07745 Jena, Germany \\ ${ }^{4}$ Helmholtz Institute, Fröbelstieg 3, 07743 Jena, Germany
}

(Received 26 June 2013; accepted 29 August 2013; published online 18 September 2013)

\begin{abstract}
We present a Michelson interferometer for $13.5 \mathrm{~nm}$ soft $\mathrm{X}$-ray radiation. It is characterized in a proofof-principle experiment using synchrotron radiation, where the temporal coherence is measured to be $13 \mathrm{fs}$. The curvature of the thin-film beam splitter membrane is derived from the observed fringe pattern. The applicability of this Michelson interferometer at intense free-electron lasers is investigated, particularly with respect to radiation damage. This study highlights the potential role of such Michelson interferometers in solid density plasma investigations using, for instance, extreme soft x-ray freeelectron lasers. A setup using the Michelson interferometer for pseudo-Nomarski-interferometry is proposed. (C) 2013 AIP Publishing LLC. [http://dx.doi.org/10.1063/1.4821146]
\end{abstract}

\section{INTRODUCTION}

With the advent of free-electron lasers (FELs) in the extreme ultraviolet (XUV) wavelength range (4-100 nm) a new field of ultra-fast science has been established in the last decade, ${ }^{1,2}$ and dedicated instruments were developed. ${ }^{3-5}$ Notably, FEL radiation is spatially and temporally coherent, ${ }^{6-8}$ which makes it particularly suitable for interferometric techniques. Experiments exploiting XUV interferometry could provide a sensitive measure of dielectric properties of solid density matter. ${ }^{9}$ For example, the refractive index $n$ in the $\mathrm{XUV}$ is close to unity and thus is challenging to determine by simple refraction experiments. In particular, a direct timeresolved measurement of $n$, for instance, in laser-driven dense plasmas requires coherent XUV radiation combined with interferometric approaches. ${ }^{10,11}$ Such measurements would improve the understanding of radiation transport in dense plasmas and warm dense matter. Therefore, the results would influence fields as diverse as inertial confinement fusion, planetary physics, and dense plasma physics. In this article, the performance of an XUV Michelson interferometer and its applicability to measure dielectric properties of solid density matter using FELs is investigated.

\section{INTERFEROMETRY CONCEPTS}

The key component of any interferometer is a beam splitter, allowing for propagation of two separated beams over a defined path length, and finally their superposition on a detector. Beam splitting is either realized by separating the beam profile geometrically in two halves (wavefront division) or by a superposition of the entire beam (amplitude division). ${ }^{12}$ In

a) vinzenz.hilbert@uni-jena.de the XUV, wavefront division is achieved by total external reflection (e.g., using Fresnel bi-mirrors ${ }^{13,14}$ ) and thus can be operated in a wide spectral range. Alternative approaches use zone plates ${ }^{15}$ or phase-shifting point diffraction. ${ }^{16}$ However, a minimum of spatial coherence is required to achieve interference. This potential drawback is overcome in amplitude dividing interferometers whose decisive advantage is their capability of generating two identical wavefront replica. ${ }^{17}$ Hence, only the latter method allows for a complete disentanglement of spatial and temporal coherence. Amplitude division is realized, e.g., by the use of separate diffraction orders of a grating ${ }^{17}$ or by thin-film beam splitters. Using the latter, Mach-Zehnder ${ }^{18}$ and Michelson-type ${ }^{19}$ interferometers have been realized in the XUV regime. In particular, the compact Michelson scheme permits independent adjustment of either path length. Based on this, longitudinal coherence measurements of $\mathrm{x}$-ray lasers ${ }^{20,21}$ and XUV Fourier-transform spectroscopy ${ }^{22,23}$ have successfully been demonstrated.

\section{EXPERIMENTAL SETUP}

In the following we describe the setup of the Michelson interferometer designed for experiments at the Free-electron LASer in Hamburg (FLASH). The interferometer is designed for a wavelength of $13.5 \mathrm{~nm}$ where FLASH operates stably and most efficiently.

\section{A. The beam splitter}

At XUV wavelengths, the design of an interferometer is particularly demanding. For a transmissive beam splitter, strong absorption and low reflectivity in the XUV have to be accounted for. Here the beam splitter consists of a $10 \mathrm{~mm}$ $\times 10 \mathrm{~mm}$ silicon frame with a free standing silicon nitride 




(a)

(b)

FIG. 1. (a) Layer composition scheme of the beam splitter. Bilayer thickness: $(9.890 \pm 0.020) \mathrm{nm}$ (Mo $3.46 \mathrm{~nm}$, Si $6.43 \mathrm{~nm})$. (b) The interferometer setup at the synchrotron source. S1 - monochromator exit slit, TM - toroidal mirror, FP - focal point, $I_{1}$ - intensity from arm with initial forward reflection, $I_{2}-$ intensity from arm with initial transmission and rear side reflection, $\mathrm{M}$ - multilayer mirrors - not to scale.

$\left(\mathrm{Si}_{3} \mathrm{~N}_{4}\right)$ membrane of $4 \mathrm{~mm} \times 4 \mathrm{~mm}$ (from Silson Ltd., USA). The thickness was measured to be $(142.0 \pm 0.5) \mathrm{nm}$ using $\mathrm{X}$-ray reflectometry. Four molybdenum-silicon $(\mathrm{Mo} / \mathrm{Si})$ bilayers were coated onto one side of the membrane (see scheme in Fig. 1(a)) for reflection at $45^{\circ}$, which is close to Brewster's angle. Therefore, reflection of p-polarized radiation is suppressed and the beam splitter has to be operated in spolarization at FEL sources which are typically linearly polarized. In the following, the beam splitter front side refers to the $\mathrm{Mo} / \mathrm{Si}$ side, whereas the membrane side will be called the rear side. Figure 2 shows calculations of the reflectivity for spolarized light for front side $(21.1 \%$ at $13.5 \mathrm{~nm})$ and rear side illumination $(1.1 \%$ at $13.5 \mathrm{~nm}$ ) for a beam splitter, accounting for tabulated absorption and reflection values. ${ }^{24}$ As compared to these, measurements (carried out at the "Physikalisch Technische Bundesanstalt") yield a slightly lower reflectivity of $R_{s-p o l}=(16.7 \pm 0.3) \%$. The transmission of the beam splitter is $T_{s-p o l}=(17.2 \pm 0.5) \%$ and therefore of the same order as the reflection.

While reflection from the front side takes place directly in the multilayer, reflection from the rear side additionally traverses the membrane twice. Due to the absorption in the membrane, the rear side reflectivity is lower than the front

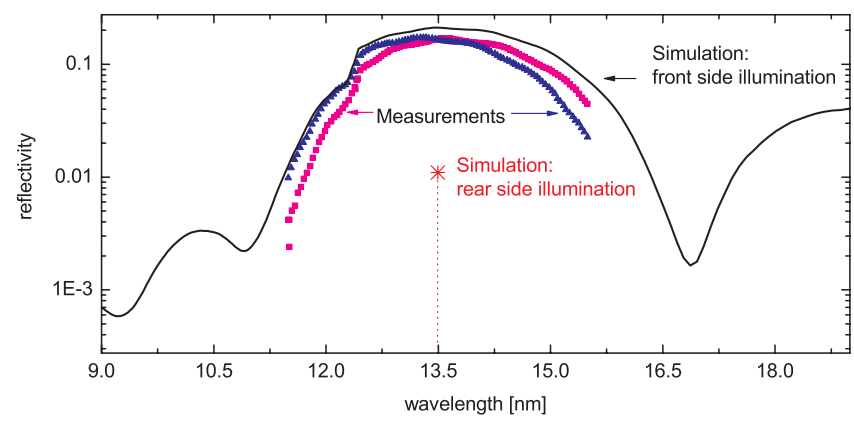

FIG. 2. Blue and magenta data show measurements for two beam splitters both with incoming light from the multilayer side (front side illumination). Differences in the spectral reflectivity can be attributed to deviations from the optimum layer thickness. The black curve shows the calculated reflectivity for front side illumination. For light impinging from the membrane side the reflectivity is calculated only for $13.5 \mathrm{~nm}$ (red data point). The decrease by one order of magnitude can be attributed to the membrane thickness and absorption, respectively. side reflectivity. Using this beam splitter in a Michelson interferometer would result in an intensity difference between the two arms, and consequently in a reduced fringe contrast. Therefore, the transmission of the brighter arm was adjusted by a $\mathrm{Zr}$ foil of $250 \mathrm{~nm}$ thickness (see setup in Fig. 1(b)), which improved the intensity ratio to $I_{1} / I_{2} \approx 1.2$. As the thicknesses of the $\mathrm{Zr}$-foil and the membrane are known, the ratio of the intensity in both arms can be used to infer the membrane density: $\varrho=(2.76 \pm 0.1) \mathrm{g} / \mathrm{cm}^{3}$. This corresponds to $(80 \pm 3) \%$ of the tabulated value for bulk material ${ }^{24}$ in agreement with previously published results. ${ }^{25}$

\section{B. Components of the Michelson interferometer}

Alignment accuracy and overall stability have to be accomplished at fractions of a wavelength, which calls for few$\mathrm{nm}$ positioning along with vibrational and thermal stability. To avoid vibrations, all optical elements are mounted on short posts fixed to a common stable base plate (see Fig. 3). Highprecision positioning of the variable arm is achieved by a tiptilt and translation piezo stage (Piezosystem Jena). A tilting angular range of $\pm 1 \mathrm{mrad}$ and a translation range of $400 \mu \mathrm{m}$



FIG. 3. Photograph of the interferometer setup. In order to distinguish the contributions from the two arms, we place a filter revolver in front of $M_{1}$, which allows blocking of this arm. The CCD detector is placed on the topside (not displayed in the image). 
can be utilized. Multilayer mirrors are placed at the end of either interferometer arm. They provide a reflectivity of $R>65 \%$ in a bandwidth of $(13.50 \pm 0.05) \mathrm{nm}$ at perpendicular incidence. As a detector, we employed a cooled Andor iKon CCD with $2048 \times 2048$ pixels of $13.5 \times 13.5 \mu \mathrm{m}^{2}$ size.

\section{PROOF OF PRINCIPLE EXPERIMENT}

A comprehensive test of the components was carried out at the synchrotron source DORIS at beamline BW $3^{26}$ (DESY Hamburg, Germany). For the experiment synchrotron radiation at $(92 \pm 0.2) \mathrm{eV}(13.5 \mathrm{~nm})$ was chosen and provided by a plane grating monochromator with a variable exit slit. At this energy the photon flux is of the order of $10^{10}$ photons/s. The interferometer setup was placed in vertical orientation, with the detector pointing upwards, to account for the s-polarization of the incoming beam. By placing the interferometer setup approximately $60 \mathrm{~cm}$ downstream of the beamline focus (see Fig. 1(b)), the beam splitter is entirely illuminated in vertical direction, whereas in horizontal direction only $1.3 \mathrm{~mm}$ of the beam splitter is illuminated. Figure 6(a) shows a typical interference pattern.

\section{A. Temporal coherence measurements}

The temporal coherence of the synchrotron radiation can be measured by analyzing the visibility of the interference pattern as a function of the difference between the two arm lengths. ${ }^{6}$ The visibility is defined as

$$
V=\frac{I_{\max }-I_{\min }}{I_{\max }+I_{\min }}
$$

with $I_{\max }$ and $I_{\min }$ being the maximum and minimum intensity of the fringe pattern, respectively.

Figure 4 shows the temporal coherence measurement. The minimum integration time of our detector amounts to $17 \mathrm{~ms}$, while synchrotron radiation constantly impinges on the interferometer. During this time, the position of piezoactuated mirror may drift slightly which potentially reduces the fringe contrast. While this problem can be overcome for

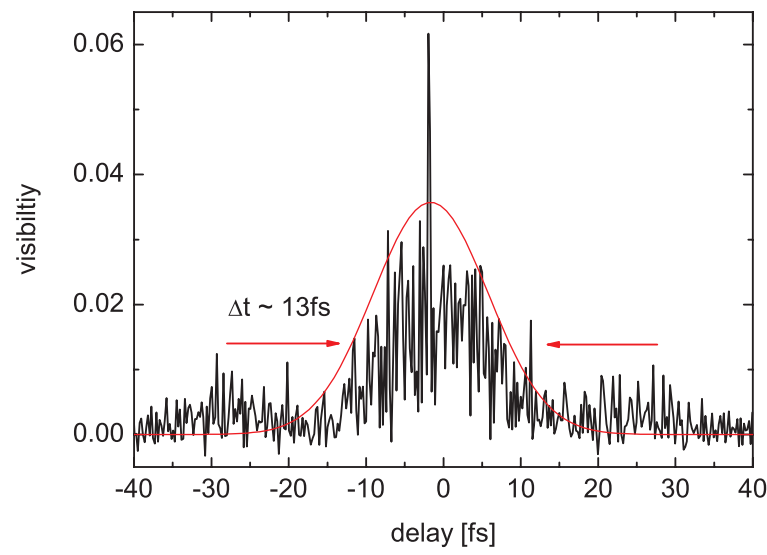

FIG. 4. Visibility as a function of delay time between the two interferometer arms. The red line is an envelope of the maximum visibility values to guide the eye. The rather low visibility is attributed to a drift of the piezo-actuated mirror in one arm during the acquisition time (17 ms).



FIG. 5. Study of surface curvature by an interference comparator at $\lambda=541.6 \mathrm{~nm}$. The stripe pattern arises from interference between an optical flat and the beam splitter. In the image, the pink square indicates the position of the membrane. The interference pattern is flat within the multilayer area, thus the flatness is better than the detection limit of about $\lambda / 4 \approx 150 \mathrm{~nm}$.

cw-sources by the use of feedback stabilized actuators, for single shot FEL pulses this does not limit the performance due to negligible movements during the ultra-short pulses. Nevertheless, in this experiment the overall envelope of the data (red line in Fig. 4) allows to determine a coherence time of about $13 \mathrm{fs}$, which is $65 \%$ of the bandwidth-limited coherence time of $20 \mathrm{fs}$ (determined by $\Delta E / E=0.02 \%$ after the monochromator).

\section{MEMBRANE SURFACE CURVATURE}

In order to determine an upper limit of the surface curvature, we measure interference fringes from optical light between an optical flat and the beam splitter. As can be seen from Fig. 5, the flatness is better than the detection limit of about $150 \mathrm{~nm}$. Figure 6(a) shows a typical interference pattern with matched arm lengths and minimized fringes. The remaining pattern can be attributed to the membrane surface curvature. In a Michelson interferometer the two contributing wavefronts stem from reflections from opposing sides of the membrane, i.e., from surfaces with opposing curvature signs. To reproduce the observed interference pattern, ray tracing simulations have been performed using the experimental parameters. We find that a quadratic function for the curvature yields sufficient agreement with the observations. The left and center images in Fig. 6(b) show the measured and calculated interference pattern, respectively. Good agreement has been achieved, which results in the 3D shape of the beam splitter shown in Fig. 6(c). The peak-to-valley amplitude amounts to $150 \mathrm{~nm}$, which is in good agreement with both the number of observed fringes and the measurements from Fig. 5.

\section{APPLICATIONS AT XUV FELS}

\section{A. Damage threshold}

The high intensities of ionizing radiation provided by free-electron lasers generate plasma when interacting with a solid. ${ }^{27,28}$ To assess the transient and possible permanent changes in material properties of the beam splitter under intense free-electron-laser radiation, one-dimensional radiation hydrodynamics simulations using HELIOS-CR ${ }^{29}$ have been 


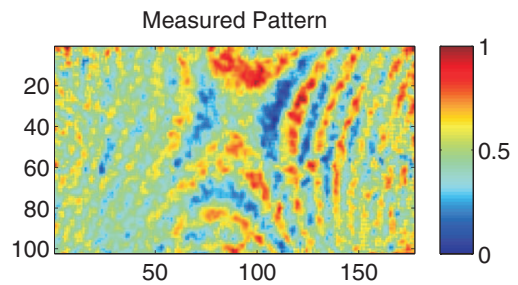

(a)

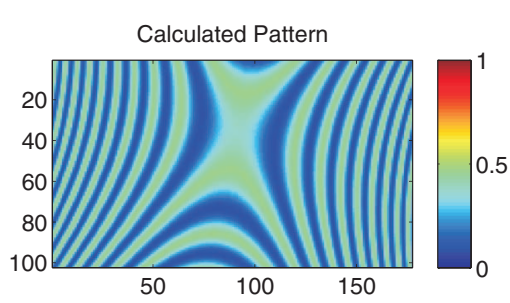

(b)

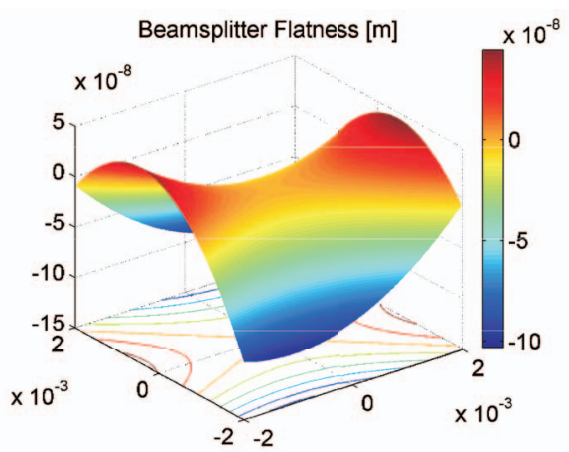

(c)

FIG. 6. (a) A typical measured interference pattern with a size of $2.4 \mathrm{~mm} \times 1.4 \mathrm{~mm}$. The raw signal is divided by a background (BGR) subtracted reference (REF) image of the XUV intensity profile, taken with unequal arm lengths $(\mathrm{T}=100 \% \times($ Signal $-\mathrm{BGR}) / \mathrm{REF})$. The transmittance value $\mathrm{T}$ (in $\%$ ) is independent of any inhomogeneous illumination. Values of $\mathrm{T}$ higher or lower than $100 \%$ (red or blue) correspond to increased and decreased intensity due to interference, respectively. (b) The best-matching calculated fringe pattern obtained with a wavefront curvature of the beam splitter. (c) Calculated surface profile of the beam splitter.

performed..$^{30}$ We model the multilayer structure and the underlying membrane, and start the simulation at ambient temperature $(25 \mathrm{meV})$. Hydrodynamic motion is suppressed for ion temperatures below the melting point, which amounts to $1683 \mathrm{~K}(0.145 \mathrm{eV})$ and $2896 \mathrm{~K}(0.25 \mathrm{eV})$ for $\mathrm{Si}$ and Mo, respectively. The sublimation point of $\mathrm{Si}_{3} \mathrm{~N}_{4}$ is at about $2200 \mathrm{~K}$. A typical irradiation scenario at FLASH is estimated as follows: using the beamline focusing mirrors, we expect a 1.5 $\times 1.5 \mathrm{~mm}^{2}$ spot at $1 \mathrm{~m}$ downstream the focus. Assuming typical parameters $(50 \mathrm{fs}, 100 \mu \mathrm{J})$ at $\lambda=13.5 \mathrm{~nm}$, an irradiation intensity of $0.1 \mathrm{TW} / \mathrm{cm}^{2}$ onto the beam splitter is expected. Figure 7 shows the result on a logarithmic time-scale up to $0.1 \mathrm{~s}$ after irradiation, which is the delay between two subsequent pulses at the typical $10 \mathrm{~Hz}$ pulse repetition rate. The FEL pulse impinges onto the multilayer structure from the left at a simulation time of $100 \mathrm{fs}$. The left graph shows the free-electron density, which rises dominantly in the first two Mo layers up to a few $10^{19} \mathrm{~cm}^{-3}$ within the pulse duration. On the same time scale, the electron temperature reaches
$0.5 \mathrm{eV}$ in the first Mo layers, while the $\mathrm{Si}$ and $\mathrm{Si}_{3} \mathrm{~N}_{4}$ components absorb less energy and stay at electron temperatures around $0.1 \mathrm{eV}$. Energy transfer to the ions and atoms is accomplished after a few tens of picoseconds, and hence the ion temperature (right graph) reaches a maximum of $70 \mathrm{meV}$ at $1 \mathrm{~ns}$ after irradiation at the boundary between the first $\mathrm{Mo} / \mathrm{Si}$ layer. This gives rise to a maximum temperature of $800 \mathrm{~K}$, well below the melting point. After $1 \mathrm{~ms}$ the beam splitter shows an evenly distributed heat profile and has cooled down to nearly initial conditions. We thus may claim that the amount of deposited energy is not sufficient to melt and destroy the beam splitter even in presence of a moderate-intense FEL pulse. A pulse back-reflected from one of the interferometer mirrors has at least a propagation distance of $2 \times 15 \mathrm{~mm}$ or a propagation time of 100 ps before it reaches the beam splitter again, and from Fig. 7 we infer that the free-electron density already dropped to its initial value after this time. The damage threshold was determined by simulations to be about $18 \mathrm{~mJ} / \mathrm{cm}^{2}$ (or $0.35 \mathrm{TW} / \mathrm{cm}^{2}$ at $50 \mathrm{fs}$ pulse duration and $350 \mathrm{~mJ}$ pulse
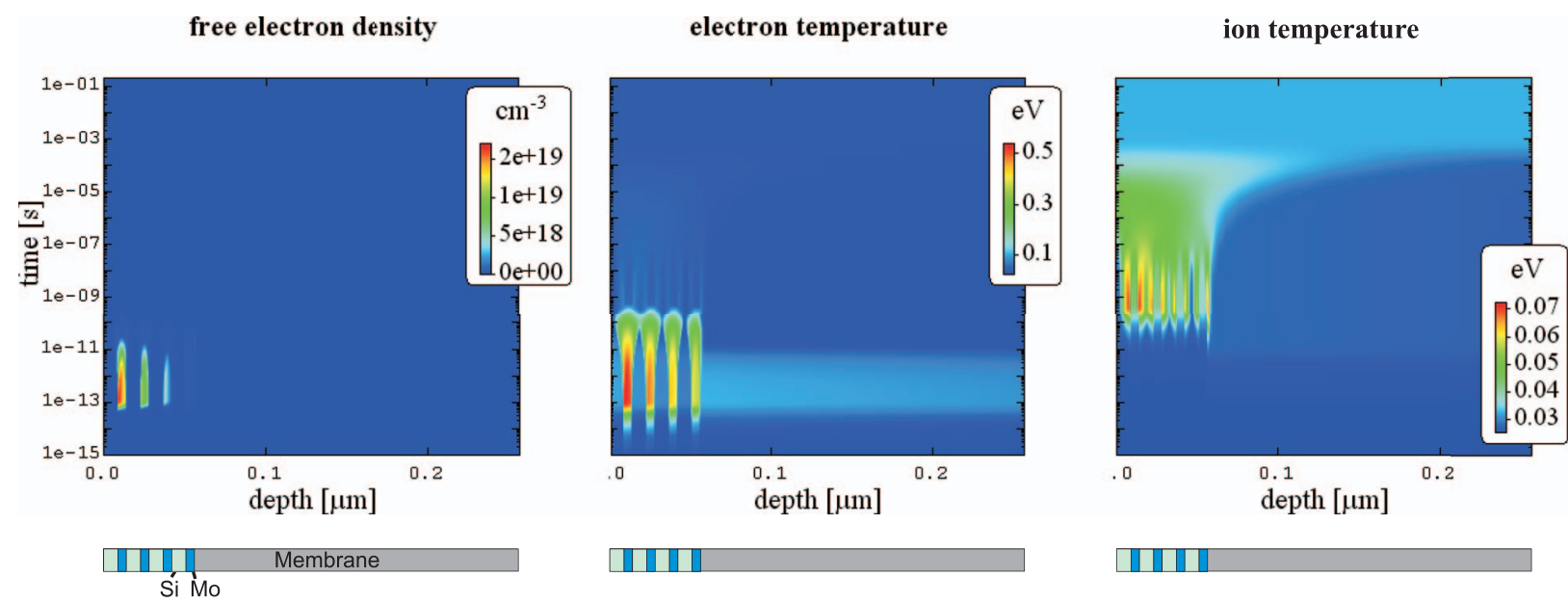

FIG. 7. Results of the hydrodynamics simulation using HELIOS-CR. A $50 \mathrm{fs}, 100 \mu \mathrm{J}$ pulse with $13.5 \mathrm{~nm}$ wavelength and $0.1 \mathrm{TW} / \mathrm{cm}^{2}$ impinges onto the multilayer structure from the left. Note the logarithmic ordinate (time) and linear abscissa (space). The free-electron density (left image) rises for a few tens of picoseconds mainly in the surface layers. The electron temperature (center image) in the multilayers rises during the first tens of picoseconds before the electrons thermalize with the ions. This can be seen in a delayed rise of the ion temperature (right image). 

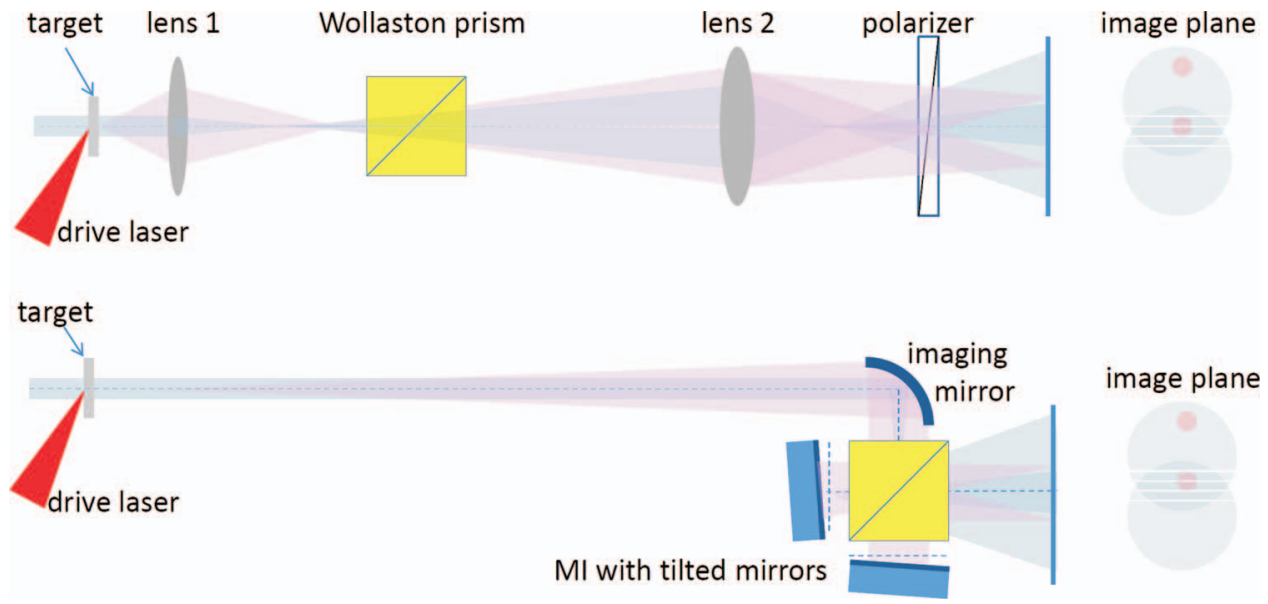

FIG. 8. Nomarski-approaches for determining the free-electron density in plasmas. A comparison of the standard optical Nomarski interferometry (top) as demonstrated in Ref. 32 and the proposed XUV pseudo-Nomarski-interferometry (bottom) is given. The blue rays illustrate the laser beams, while light red refers to the imaging properties of the optics.

energy). Above, the ion temperature in the Si layers exceeds the melting point and the multilayer structure is likely to be deteriorated . Experimentally, crater formation in $\mathrm{Mo} / \mathrm{Si}$ multilayers was observed ${ }^{31}$ at irradiation $>(45 \pm 7) \mathrm{mJ} / \mathrm{cm}^{2}$ for comparable experimental conditions. We note that our hydrodynamics simulations apply in general for all multilayer mirrors used in the interferometer, and further for any multilayer mirror placed in a comparably intense FEL beam of any experiment. It should also be considered that diffusion rates between the Mo and Si layers are increased due to the elevated temperature during and shortly after the intense FEL irradiation. After extensive use, the multilayer structure may thus show a reduced performance.

\section{B. Pseudo-Nomarski XUV interferometry}

Finally, we propose to use the XUV Michelson interferometer downstream of a target configuration to image the phase changes in strongly-driven dense matter. The proposed setup is an analog to Nomarski interferometry ${ }^{33}$ commonly employed in visible-light optics, which relies on spatially coherent illumination. This scheme can be used for the time-resolved observation of plasma plumes expanding from laser-irradiated solids. ${ }^{32,34}$ Since the critical density $n_{c}=\omega^{2} \epsilon_{0} m / e^{2}$ for XUV radiation is greater than $10^{24} \mathrm{~cm}^{-3}$, our method allows studying solid-density matter, while for optical light propagation is limited to free-electron densities of $n_{c}<10^{21} \mathrm{~cm}^{-3}$. Here, $\omega$ is the laser angular frequency, $\epsilon_{0}$ is the vacuum permittivity, and $e$ is the electron charge. A common Nomarski-type interferometry scheme is shown in the top part of Fig. 8. A collimated optical probe laser pulse (blue) is transmitted through a laser-driven target. The target plane is imaged by a set of magnifying lenses. A Wollaston prism separates the ordinary and extra-ordinary components by a small angular offset. The two radiation cones still overlap partially in the detector plane, but their polarization is perpendicular to each other. In order to achieve interference, a polarizer has to be introduced. In the XUV regime, we propose to replace the imaging lenses by an imaging mirror, as shown in the bot- tom part of Fig. 8. The unfocused FLASH beam (blue) acts as a probe. We use the Michelson interferometer in analogy to the Wollaston-prism, to overlap undisturbed wavefronts with neighboring distorted ones which alters the fringe pattern according to phase shifts. The lateral wavefront separation will be less than $1 \mathrm{~mm}$, which lies well within the spatial coherence of FLASH. ${ }^{8}$

\section{CONCLUSION}

An XUV Michelson interferometer was developed for applications at XUV FELs and has been extensively tested using $13.5 \mathrm{~nm}$ synchrotron radiation. In a proof of principle experiment, the coherence time of the synchrotron radiation was determined to be 13 fs. From the observed fringe pattern we derived the curvature of the thin film beam splitter membrane which exhibits a peak-to-valley amplitude of $150 \mathrm{~nm}$ only. Using 1D-hydrodynamics simulations assuming an irradiation flux of $0.1 \mathrm{TW} / \mathrm{cm}^{2}$, typical for FEL experiments, we derive an ion temperature well below the melting point and hence would exclude possible damage. The damage threshold was determined to be $\approx 18 \mathrm{~mJ} / \mathrm{cm}^{2}$ from simulations. In particular, this study highlights the potential role of Michelson interferometers in solid density plasma investigations. A setup for pseudo-Nomarski-interferometry at XUV FELs is proposed. Developing this technique in the XUV range opens new perspectives for the determination of transient dielectric properties of strongly driven optically opaque matter.

\section{ACKNOWLEDGMENTS}

We thank S. Zhang for help in the laboratory. Financial support by the German Federal Ministry of Education and Research (project FSP 301-FLASH), the Deutsche Forschungsgemeinschaft (Sonderforschungsbereich SFB 652), and the Volkswagen Foundation is gratefully acknowledged. The authors are greatly indebted to J. Viefhaus and L. Glaser for assistance with the BW3 beamline at DESY, as well as the staff 
of our design office and the mechanical workshop in Jena. We thank P. Zeitoun and R. Sobierajski for fruitful discussions.

${ }^{1}$ W. Ackermann et al., Nature Photon. 1, 336 (2007).

${ }^{2}$ E. Allaria, C. Callegari et al., New J. Phys. 12, 075002 (2010).

${ }^{3}$ R. R. Fäustlin, U. Zastrau et al., J. Instrum. 5, P02004 (2010).

${ }^{4}$ S. Fuchs, C. Rödel et al., Rev. Sci. Instrum. 84, 023101 (2013).

${ }^{5}$ U. Zastrau, V. Hilbert et al., J. Instrum. 6, P10001 (2011).

${ }^{6}$ W. F. Schlotter, F. Sorgenfrei et al., Opt. Lett. 35, 372 (2010).

${ }^{7}$ A. Singer, I. A. Vartanyants et al., Phys. Rev. Lett. 101, 254801 (2008).

${ }^{8}$ S. Roling, B. Siemer et al., Phys. Rev. ST Accel. Beams 14, 080701 (2011)

${ }^{9}$ L. B. D. Silva, J. T. W. Barbee et al., Appl. Opt. 34, 6389 (1995).

${ }^{10}$ P. Zeitoun, M. Fajardo, and G. Lambert, Nature Photon. 4, 739 (2010).

${ }^{11}$ L. Da Silva, T. Barbee et al., Rev. Sci. Instrum. 66, 574 (1995).

${ }^{12}$ B. Born and E. Wolf, Principles of Optics (Cambridge University Press, 1999).

${ }^{13}$ F. Albert, P. Zeitoun et al., Opt. Commun. 142, 184 (1997).

${ }^{14}$ J. Svatos, D. Joyeux et al., Opt. Lett. 18, 1367 (1993).

${ }^{15}$ M. Wieland, C. Spielmann et al., Appl. Phys. B 76, 885 (2003).

${ }^{16} \mathrm{H}$. Medecki, E. Tejnil et al., Opt. Lett. 21, 1526 (1996).
${ }^{17}$ J. Filevich, K. Kanizay et al., Opt. Lett. 25, 356 (2000).

${ }^{18}$ P. Celliers, J. C. Moreno et al., Opt. Lett. 20, 1907 (1995).

${ }^{19}$ A. Morlens, P. Zeitoun et al., Laser Part. Beams 22, 279 (2004).

${ }^{20}$ R. Smith, F. Delmotte et al., Opt. Lett. 28, 2261 (2003).

${ }^{21}$ J. Dunn, F. Delmotte et al., Proc. SPIE 5197, 43 (2003).

${ }^{22}$ P. Zeitoun, P. Balcou et al., Appl. Phys. B 78, 983 (2004).

${ }^{23}$ P. Zeitoun, P. Balcou et al., Proc. SPIE 5197, 194 (2003).

${ }^{24}$ See http://henke.lbl.gov for the center for x-ray optics (2012).

${ }^{25}$ F. Delmotte, M. F. Ravet et al., Appl. Opt. 41, 5905 (2002).

${ }^{26}$ O. Bjorneholm, F. Federmann et al., Rev. Sci. Instrum. 66, 1732 (1995).

${ }^{27}$ B. Nagler, U. Zastrau et al., Nat. Phys. 5, 693 (2009).

${ }^{28}$ U. Zastrau, C. Fortmann et al., Phys. Rev. E 78, 066406 (2008).

${ }^{29}$ J. MacFarlane, I. Golovkin, and P. Woodruff, J. Quant. Spectrosc. Radiat. Transf. 99, 381 (2006)

${ }^{30}$ J. D. Johnson and S. Lyon, SESAME 2984 (Los Alamos National Laboratory, 1997).

${ }^{31}$ A. Khorsand, R. Sobierajski et al., Opt. Express 18, 700 (2010).

${ }^{32}$ L. Cao, I. Uschmann et al., Laser Part. Beams 25, 239 (2007).

${ }^{33}$ G. Nomarski, J. Phys. Radium 16, S9 (1955).

${ }^{34}$ R. Benattar, C. Popovics, R. Sigel et al., Rev. Sci. Instrum. 50, 1583 (1979). 